\title{
Filling gaps in notification data: a model- based approach applied to travel related campylobacteriosis cases in New Zealand
}

\author{
E. Amene ${ }^{1 *}$, B. Horn² ${ }^{2}$ R. Pirie², R. Lake ${ }^{2}$ and D. Döpfer ${ }^{1}$
}

\begin{abstract}
Background: Data containing notified cases of disease are often compromised by incomplete or partial information related to individual cases. In an effort to enhance the value of information from enteric disease notifications in New Zealand, this study explored the use of Bayesian and Multiple Imputation (MI) models to fill risk factor data gaps. As a test case, overseas travel as a risk factor for infection with campylobacteriosis has been examined.
\end{abstract}

Methods: Two methods, namely Bayesian Specification (BAS) and Multiple Imputation (MI), were compared regarding predictive performance for various levels of artificially induced missingness of overseas travel status in campylobacteriosis notification data. Predictive performance of the models was assessed through the Brier Score, the Area Under the ROC Curve and the Percent Bias of regression coefficients. Finally, the best model was selected and applied to predict missing overseas travel status of campylobacteriosis notifications.

Results: While no difference was observed in the predictive performance of the BAS and MI methods at a lower rate of missingness $(<10 \%)$, but the BAS approach performed better than $\mathrm{Ml}$ at a higher rate of missingness (50\%, $65 \%, 80 \%)$. The estimated proportion (95\% Credibility Intervals) of travel related cases was greatest in highly urban District Health Boards (DHBs) in Counties Manukau, Auckland and Waitemata, at $0.37(0.12,0.57)$, $0.33(0.13,0.55)$ and $0.28(0.10,0.49)$, whereas the lowest proportion was estimated for more rural West Coast, Northland and Tairawhiti DHBs at $0.02(0.01,0.05), 0.03(0.01,0.08)$ and $0.04(0.01,0.06)$, respectively. The national rate of travel related campylobacteriosis cases was estimated at $0.16(0.02,0.48)$.

Conclusion: The use of BAS offers a flexible approach to data augmentation particularly when the missing rate is very high and when the Missing At Random (MAR) assumption holds. High rates of travel associated cases in urban regions of New Zealand predicted by this approach are plausible given the high rate of travel in these regions, including destinations with higher risk of infection. The added advantage of using a Bayesian approach is that the model's prediction can be improved whenever new information becomes available.

Keywords: Campylobacteriosis, Bayesian specification, Multiple imputation, Missing value

Abbreviations: AUC, Area under the curve; BAS, Bayesian specification, BS, Brier score; CC, Complete case; $\mathrm{Cl}$, Credibility interval; DI, Deprivation index; ESR, Environmental sciences research; MAR, Missing at random; MCAR, Missing completely at random; MCMC, Markov Chain Monte Carlo; MI, Multiple imputation; MICE, Multiple imputation using chained equations; MNAR, Missing not at random; PB, Percent bias; SD, Standard deviation

\footnotetext{
* Correspondence: amene@wisc.edu

${ }^{1}$ Department of Medical Sciences, School of Veterinary Medicine, University

of Wisconsin-Madison, Madison, USA

Full list of author information is available at the end of the article
} 


\section{Background}

Information originating from investigation of notified cases of an infectious disease has the potential to inform about the epidemiology and risk factors associated with the disease. Aggregating demographic and risk factor information from surveillance systems can help to set policy, monitor trends, and develop risk management options. However, the value of this information is often compromised by incomplete or partial information related to individual cases.

In New Zealand, cases of notifiable diseases are reported by general practitioners, laboratories and public health workers and the information is stored in the EpiSurv database. EpiSurv is operated by the Institute of Environmental Science and Research (ESR) on behalf of the Ministry of Health. A series of case report forms (https://surv.esr.cri.nz/episurv/index.php) are used to collect information about cases, disease diagnosis and clinical course, risk factors for the disease and case management.

Campylobacteriosis has been a notifiable disease in New Zealand since 1980. Data from notified cases are reported annually in surveillance summaries and have been analyzed for trends and to assess the effect of specific interventions $[1,2]$. These analyses are primarily based on demographic information, since for a variety of reasons the risk factor information is not supplied for all cases. However, the value of complete information on cases has been demonstrated by a sentinel site study in the Manawatu region of New Zealand, which has made a special effort to complete risk factor reporting, alongside microbial subtyping [3].

In an effort to enhance the value of information from campylobacteriosis notifications in New Zealand, we have explored the use of models to fill risk factor data gaps. As a test case, we examined overseas travel as a risk factor for campylobacteriosis. Identifying the proportion of cases of campylobacteriosis where infection was acquired overseas is important to properly understand and measure domestic risk factors and the success of any risk management interventions [4]. International travel as a risk factor is important, as the rate of overseas travel by New Zealanders is high (e. g. 46 trips per 100 per year as compared to the international average of 14 per 100 in 2008) [5, 6]. However, whether (or not) cases had travelled overseas as a potential risk factor is reported for less than half of the notified cases of campylobacteriosis, and the reporting of this factor varies considerably across the 20 District Health Boards (DHBs) in New Zealand. One approach to adjusting for this lack of data, as currently used in annual surveillance reports, is to apply the proportion travel related from the campylobacteriosis cases for which the information is available to those cases lacking travel information.
This approach estimates that approximately $7 \%$ of campylobacteriosis notifications nationally over the period 2000 to 2010 were acquired overseas. However this information may be biased and does not fully reflect regional variation. As an alternative, we applied Multiple Imputation (MI) [7] and Bayesian Specification (BAS) [8] models, seeking to adjust rates of travel associated illness and fill data gaps using covariates derived from demographic characteristics and travel rates in the general New Zealand population.

\section{Methods \\ Empirical data \\ Campylobacteriosis notifications}

Campylobacteriosis notification records were obtained from the EpiSurv database [9]. All case notifications were completely anonymized to conceal the identity of individuals. The database registers a number of demographic and risk factor characteristics of the cases in addition to clinical features. Regional information is available per DHB in the campylobacteriosis notification data.

There were 121,764 notifications of campylobacteriosis in New Zealand reported between 2000 and 2010. Of these, most were culture confirmed ('Confirmed') or epidemiologically linked to confirmed cases or outbreak sources ('Probable') (Table 1). As there are no definitive results for the cases with a case status of 'Under investigation' and 'Unknown', we excluded them from the analysis resulting 119,375 cases for the primary dataset (sum of the first two columns in Table 1). Among 119,375 cases, 44,285 (37.1\%) had complete information for the travel section of the EpiSurv questionnaire, and 3107 (7\%) of cases with information for this section had completed short term international travel. Since $0.6 \%$ of Age and $1.6 \%$ of Sex observations were missing in the primary dataset, the associated records were excluded making the total number of cases available for analysis to become 116,721. The dependent variable used for our regression model was overseas travel status of the notified cases.

Table 1 Total number of campylobacteriosis notification in New Zealand residents categorized by information on overseas travel (2000-2010)

\begin{tabular}{llllll}
\hline Travel status & \multicolumn{5}{l}{ Campylobacteriosis status } \\
\cline { 2 - 6 } & Confirmed & Probable & $\begin{array}{l}\text { Under } \\
\text { investigation }\end{array}$ & Unknown & Total \\
\hline No & 41617 & 60 & 52 & 416 & 42145 \\
Unknown & 74481 & 110 & 222 & 1653 & 76466 \\
Yes & 3100 & 7 & 7 & 39 & 3153 \\
Total & 119198 & 177 & 281 & 2108 & 121764 \\
\hline
\end{tabular}




\section{Explanatory variables}

A number of explanatory variables was derived from the EpiSurv and Statistics New Zealand databases to construct a regression models for predicting missing travel status of notified campylobacteriosis cases. A complete list of predictor variables extracted from the notification and travelers' database is shown in Table 2 and a detailed description is given in Additional file 1. While Deprivation index, Urban (population under urban influence) and Travel Rate are variables at a District Health Board (=DHB) level; Age, Sex, Season and Intervention (whether the case was recorded before or after 2006) are case specific variables.

\section{Statistical methods}

\section{Logistic regression}

Since our response variable (overseas travel status) is a binary variable $(1=$ yes, $0=\mathrm{No})$, a logistic regression model was applied to the data. The generalized form of the logistic regression model is shown in eq. (1).

$$
\log \left(\frac{p(Y=1)}{1-p(Y=1)}\right)=\beta_{0}+\sum_{j=1}^{k} \beta_{j} x_{j}
$$

Where $p(Y=1)$ is the probability that a case made short term overseas travel and $\beta$ 's are the regression coefficients, $k=$ number of covariates ( $x$ 's). See Table 2 and Additional file 1 for the detail description of the covariates.

Table 2 Description of variables in the New Zealand campylobacteriosis notification and short term international travelers' datasets (2000-2010)

\begin{tabular}{|c|c|}
\hline Variables & Details \\
\hline $\begin{array}{l}\text { Deprivation } \\
\text { index }\end{array}$ & $\begin{array}{l}\text { Categorical, } 1-10 \text { scale ( } 1=\text { least deprived, } 10=\text { most } \\
\text { deprived) }\end{array}$ \\
\hline Urban & $\begin{array}{l}\text { Numeric, Proportion of DHB population under urban } \\
\text { influence }\end{array}$ \\
\hline $\mathrm{DHB}$ & Categorical, Residence District Health Board \\
\hline Travel rate & $\begin{array}{l}\text { Numeric, Residence DHB's rate of short term } \\
\text { international travel }\end{array}$ \\
\hline Report date & Year of campylobacteriosis notification, 2000-2010 \\
\hline Age & Four categories; $<5,5-19,20-65$ and $65+$ Years \\
\hline Sex & Two categories; Male and Female \\
\hline Season & $\begin{array}{l}\text { Four categories; Spring (Sep-Nov), Summer (Dec-Feb), } \\
\text { Autumn (Mar-May) \& Winter (Jun-Aug) }\end{array}$ \\
\hline Overseas travel & $\begin{array}{l}\text { Three categories; Yes, No, Unknown ( } 62 \% \text { of the cases } \\
\text { did not have travel information.) }\end{array}$ \\
\hline Intervention & $\begin{array}{l}\text { A binary indicator variable to identify before and after } \\
\text { the } 2006 \text { poultry intervention period. }\end{array}$ \\
\hline
\end{tabular}

Notes: Deprivation index, Urban, DHB and Travel Rate are DHB level variables, whereas Report Date, Age, Season, Overseas Travel and Intervention are measured at an individual case level
Missing At Random Assumption (MAR) There are three types of missing data mechanisms, namely Missing Completely At Random (MCAR), Missing At Random (MAR) and Missing Not At Random (MNAR) [10]. MCAR occurs when the missingness is completely at random and results obtained from only completely observed cases can be used for inference, whereas MNAR indicates a systematic missingness in the data and requires explicit model for the missing data mechanism. The MAR scenario on the other hand also requires a model but can use measured covariates. MAR assumes that the probability of missingness only depends on the covariates in the data. In the MCAR and MAR situations, the missing data mechanism is frequently referred to as ignorable, i.e., we do not need a separate model for the missing data mechanism. In our case, the covariates obtained from Statistics New Zealand and the Episurv were used to construct the model. Most missing data methods including MI require this assumption to be fulfilled for a valid inference. While the MAR assumption, as such, is not statistically testable, it can be supported by demonstrating association of predictors with the missingness. We investigated this by fitting a logistic regression with dependent variable missingness of overseas travel $(1=$ missing, $0=$ otherwise $)$ on covariates. A statistically significant association indicates that the missingness can be explained by the covariates (i.e., the MAR assumption can hold.) A detailed description of types of missing data can be found in the literature $[10,11]$.

\section{Multiple imputation}

Multiple Imputation is a principled way of handling incomplete data where missing observations are replaced by draws from the predictive distribution of the missing data given the observed data [12, 13]. According to Rubin (1996), MI is a three-step process. First, sets of plausible values for missing observations are created. Each of these sets of values 'fill-in' the missing values (assuming MAR) and create multiple 'complete' datasets, so called 'multiply' datasets. Simulation studies have shown that as few as 3 'multiply' datasets are adequate for a dataset with $20 \%$ missing values [14]. Other studies have shown that 5-10 'multiply' datasets are usually optimum depending on the proportion missing [7]. Second, each of these 'multiply' datasets can be analyzed using standard complete data methods. Finally, the results are pooled using Rubin's rule, which allows the uncertainty regarding the imputation to be taken into account [15]. The $R$ package MICE (Multiple Imputation using Chained Equations) was used for performing MI [7]. In this study, we have used 20 multiply datasets. We used the pooled regression coefficients to construct a logistic regression equation for predicting the probability of overseas travel. 
All potential predictors available in our dataset were incorporated into the imputation model. Including all covariates predictive of overseas travel will help the MAR assumption to be increasingly plausible, in addition to producing unbiased results [16, 17]. This is because subjects with missing data based on (other) known characteristics, i.e. MAR- are by definition a random subset from the sample given these known characteristics (Table 1).

\section{Bayesian Specification (BAS)}

The Bayesian method allows to jointly use information coming from the observed data and from prior information on unknown parameters to derive inferences about missing data and parameters using Markov ChainMonte Carlo (MCMC) algorithm [18]. While MI was derived from within a Bayesian framework (sampling from the posterior distribution of missing values, conditional on observed values), Bayesian approaches have been applied more generally [19]. Bayesian modelling provides a flexible method for incorporating different assumptions about the missing data mechanism and accommodating different patterns of missing data in the model [20]. For example, we can specify a separate model for the missing data mechanism if the information for estimating the missingness obtained (i.e., in the case of so called informative missing response') [21]. In our case, however, the data contain no information regarding the mechanism by which missing data were introduced and therefore we assume the missing data mechanism to be ignorable. In this case, the BAS treats missing data as additional unknown parameters and automatically generates values from its posterior predictive distribution for filling the missing data.

We used the JAGS 3.4.0. program (Just Another Gibbs Sampler) for Bayesian analysis, which is called into the $\mathrm{R}$ environment through rjags package [22]. The use of a Bayesian method requires that the priors of unknown parameters to be specified properly [23]. This is a way of incorporating uncertainty about the parameters into the model. For our analysis, all regression coefficients and the intercept were assigned uninformative priors (a normal
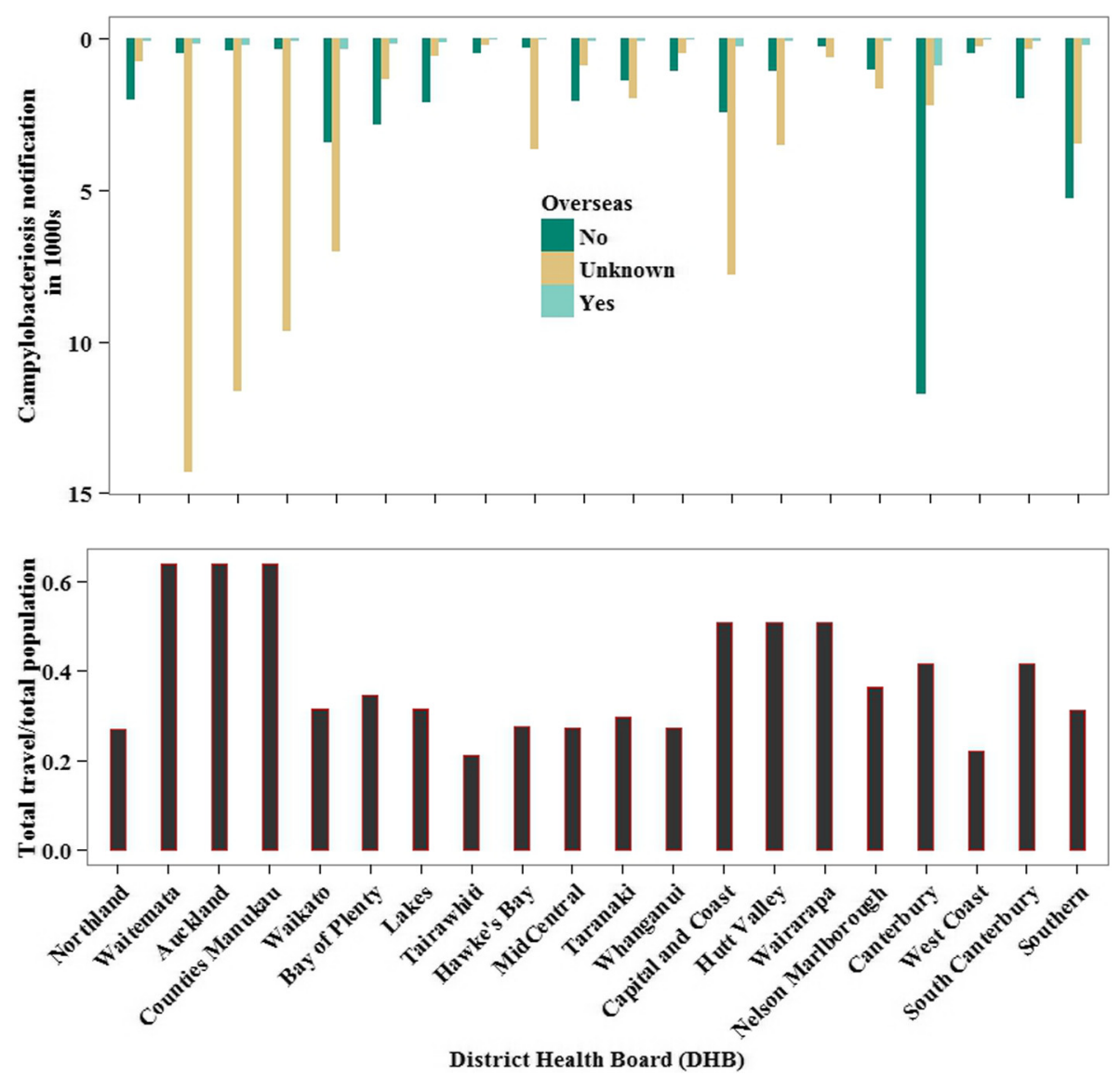

Fig. 1 Distribution of campylobacteriosis notification categorized by the status of overseas travel (upper panel) and the annual proportion of short term international travels (lower panel), in DHBs of New Zealand (2000 - 2010). Notes: Upper panel: campylobacteriosis notification in 1000s is the sum of all cases notified between 2000 and 2010 in a given District Health Board; lower panel: Total travels/total population: the average number of outbound travels per year divided by the average population size per year between 2000 and 2010 for a given District Health Board 
distribution with mean 0 and standard deviation of 100 , i.e. each with an inverse variance of $10^{-4}$ ) (Additional file 2) [8]. For computational reasons, Bayesian models in $J A G S$ require the variance to be specified in terms of the precision (inverse of the variance). The models were run for 30,000 iterations with the first 3000 iterations discarded as burn-ins. All models were initialized with two chains. For realistic starting values, we set the initial values for each chain obtained from the fitted regression coefficients (see Additional file 2). As Bayesian inference relies on MCMC algorithm to draw samples from the posterior distribution, convergence of the algorithm has to be assessed, i.e., whether the Markov chains have reached a stable equilibrium distribution. Convergence indicates that the samples from the MCMC process are, in fact, drawn from the actual joint posterior distribution of the parameters. This was done through visually evaluating density plots, autocorrelation and the Brooks-Gelman-Rubin (BGR) statistic of the parameters in the models. The BGR statistic is a convergence diagnostic that compares the within and between chain variances where a value around 1 indicates convergence [23].

\section{Data analysis \\ Model development}

First, we fit a multiple logistic regression model $(E q .2)$ to the dataset containing Complete Cases (CC) $(n=44,285)$ using Frequentist and Bayesian frameworks. The CC analysis refers to analysis restricted to campylobacteriosis notifications with fully reported travel status (i.e., disregarding missing values). This subset of the original dataset included $38 \%(44,285)$ of all notifications reported between 2000 and 2010. The remaining $62 \%(72,436)$ lack travel
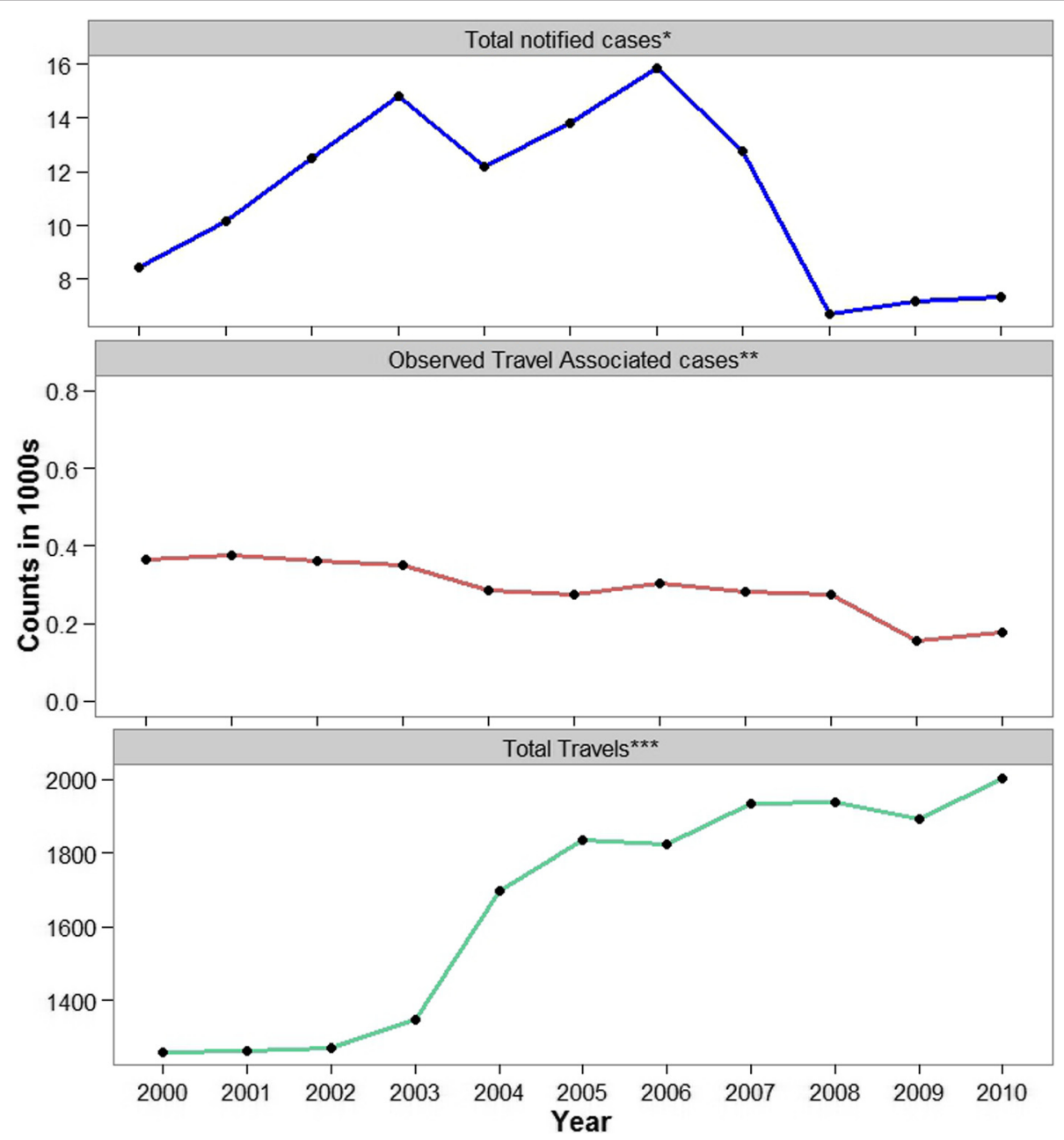

Fig. 2 Annual short term international travel and campylobacteriosis notification of New Zealand residents (2000-2010). * Total notified cases: total number of campylobacteriosis cases notified between 2000 and 2010. ${ }^{* *}$ Observed travel associated cases: campylobacteriosis cases that had confirmed overseas travel during the incubation period of the disease. ${ }^{* *}$ Total travels: total number of short term international travels between 2000 and 2010. Short term international travel is defined as international departures of New Zealand residents for an intended period of less than 12 months (Statistics New Zealand [www.stats.govt.nz]) 
information. The reason for performing this restricted analysis was to select the best prediction model based on cases with complete data.

$$
\begin{aligned}
\log \left(\frac{p(Y=1)}{1-p(Y=1)}\right) & =\beta_{0}+\beta_{1} * \text { URBAN }+\beta_{2} * \text { DEPRIVATION } \\
& +\beta_{3} * \text { TRAVEL }+\beta_{4} * \text { AGE } \\
& +\beta_{5} * \text { SEASON }+\beta_{6} * \text { SEX } \\
& +\beta_{7} * \text { INTERVENTION }
\end{aligned}
$$

Next, we investigated the performance of MI and BAS for different rates of artificially introduced missing data to the CC $(10 \%, 50 \%, 65 \%$ and $80 \%$ missingness on overseas travel status was introduced). In order to achieve the required percentage of missing values, we stratified the data into a cross tabulation based on two strata of the variable SEX and four artificial strata of the variable URBAN (i.e., $\leq 0.6=1,0.6-0.8=2,0.8-0.9=3$, $>0.9=4)$. The SEX and URBAN variables were chosen for convenience. Then, we deleted the stratum (or strata) from the cross tabulation where the counts sum up to the desired proportion of missingness. Deleting specific strata from the dataset will ensure that the resulting missing data are MAR. We generated one sample per each category of artificially introduced missing data. Then, for every category, we fit a separate logistic regression model (Eq. 2) and summarized the outputs in Fig. 4. Finally, based on the models' performance parameters on missing data prediction, we selected the best model and applied it for predicting overseas travel status in the original dataset.

\section{Model evaluation and performance}

We evaluated the performance of our models by comparing Percent Bias (PB) and Brier Score (BS) of regression coefficients and predictions, respectively. The $P B$ indicates the percent deviation of the regression coefficients of models fitted to the missing data as compared to those estimated by the fully observed dataset (i.e. Complete Cases) (Eq. 3). Note that, the description of bias used here is slightly different to the usual definition (the expectation of difference between parameter estimates) [21].

$$
\left.P B=\left(\beta_{m}-\beta_{f}\right) / \beta_{f}\right) * 100
$$

where $\beta_{f}$ is the regression coefficient estimated from the models fitted to the complete cases, and $\beta_{m}$ is the regression coefficient estimated from the other models (i.e. using data including missing values). The BS, on the other hand, is an overall measure of predictive performance, i.e. a combination of discrimination and calibration

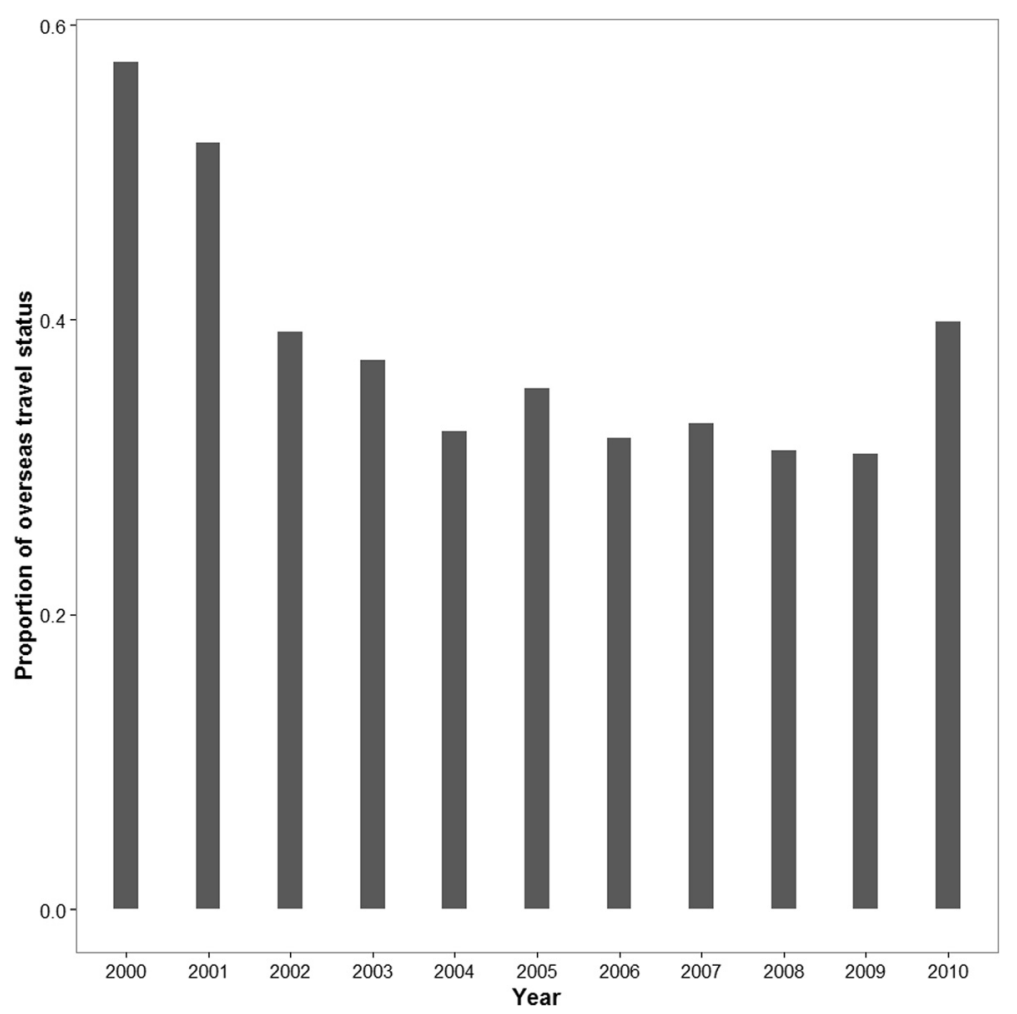

Fig. 3 The proportion of campylobacteriosis notifications in New Zealand with known status of overseas travel information (2000-2010) 
Table 3 Summary of logistic regression analysis for variables predicting missing indicator ( $1=$ missing overseas travel information, $0=$ otherwise) to test the validity of Missing At Random assumption $(n=116721)$

\begin{tabular}{lccc}
\hline Coefficients & Estimate & Std. Error & $\operatorname{Pr}(>|z|)$ \\
\hline Intercept) $^{\text {Urban }}{ }^{\mathrm{a}}$ & -8.757 & 0.089 & $<0.001$ \\
Deplndex $^{\mathrm{b}}$ & 2.992 & 0.103 & $<0.001$ \\
Travel Rate $^{\mathrm{c}}$ & 0.525 & 0.006 & $<0.001$ \\
Age (5-19) & 0.081 & 0.001 & $<0.001$ \\
Age (20-59) & 0.154 & 0.027 & $<0.001$ \\
Age (60+) & 0.033 & 0.023 & 0.145 \\
Summer & -0.142 & 0.027 & $<0.001$ \\
Autumn & 0.014 & 0.018 & 0.443 \\
Winter & -0.002 & 0.021 & 0.94 \\
Male & 0.035 & 0.021 & 0.085 \\
Intervention $^{\mathrm{d}}$ & 0.153 & 0.014 & $<0.001$ \\
\hline
\end{tabular}

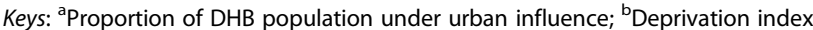
(scale $0-10,0$ being least deprived and 10 being most deprived DHB; ${ }^{\text {'S }}$ Short term international travel per 100 residents of a $\mathrm{DHB} ;{ }^{\mathrm{d}} \mathrm{A}$ binary indicator variable to identify pre and post 2006 intervention. Age $(<5)$, Spring, and Female sex are reference categories

[24] (Eq. 4). The BS, or average prediction error is defined as follows:

$$
\begin{gathered}
B S=\frac{1}{N} \sum_{i=1}^{N}\left(f_{i}-O_{i}\right)^{2} \\
i=1, \ldots, N
\end{gathered}
$$

where $f_{i}$ are predicted probabilities by the model, $O_{i}$ is the observed outcome ( 0 or 1 ), and $N$ is the total number of observations. A $B S$ value close to 0 indicates the model performs well, whereas larger scores indicate poorly fitting models [25].

Additionally, we evaluated our models using the area under the receiver operating characteristic (ROC) curve. The $R O C$ is often used to summarize and compare the discriminatory accuracy of a diagnostic test or modality, and to evaluate the predictive power of statistical models for binary outcomes [26]. We used the ROC curve analysis to evaluate how accurate our logistic regression models were in predicting overseas travel. Accordingly, we selected the $B A S$ approach as a method of choice to apply to the original dataset.

\section{Prediction of overseas travel}

A Bayesian logistic regression model was fitted to the original dataset $(n=116,721)$ to predict missing overseas travel status of notified campylobacteriosis cases. The priors for all parameters in the model were specified as uninformative (see Additional file 2). We ran the sampler for 30,000 iterations and used 2 chains and 3000 iteration burn-ins. Finally, we investigated model fit by examining density plots, autocorrelation and trace plots of a subset of parameters in the model for a visual graphical assessment. After a convergence was achieved (i.e., after each chain mixed well and appeared stationary indicating that the target distribution was reached), we extracted the predicted summary measures of probability of overseas travel for individual cases (mean and standard deviation) from the posterior distribution. Since our main interest was to produce average predictions per reporting region (DHB), we summarized those individual predictions into a pooled mean $\left(\bar{\mu}\left(x_{i}\right)\right)$ and SD per reporting region $\left(S D_{j}\right)$. To compute these values, we stratified the predicted probabilities by DHB, and then we calculated the mean (expected value) and the pooled SD per DHB, respectively, as shown in Eqs. 5 and 6 below.

$$
E\left(X_{j}\right)=\bar{\mu}=\frac{1}{n} \sum_{i=1}^{n} x_{i}
$$

where $D H B_{j}(j=1,2, \ldots, 20)$ consisting of $n$ elements $x_{1}, . ., x_{n}$ denoting individual predictions.

$$
S D_{j}=\sqrt{\frac{1}{n} \sum_{i=1}^{n}\left(x_{i}-\bar{\mu}\right)+\left(\frac{1}{n} \sum_{i=1}^{n} \sigma_{i}^{2}\right)}
$$

where $S D_{j}$ denotes the pooled SD for $D H B_{j}, x_{i}=$ individual predictions, $\bar{\mu}_{j}=$ the mean prediction for $D H B_{j}, \sigma_{i}^{2}=$ the variance of individual predictions and $n=$ number of

\begin{tabular}{|c|c|c|c|c|c|c|c|c|c|c|}
\hline \multirow{3}{*}{$\begin{array}{l}\text { Accuracy } \\
\text { measure }\end{array}$} & \multicolumn{2}{|c|}{ Complete data $^{a}$} & \multicolumn{8}{|c|}{ Missing data ${ }^{b}$} \\
\hline & \multirow[t]{2}{*}{ Frequentist } & \multirow[t]{2}{*}{ Bayesian } & \multicolumn{4}{|c|}{ Multiple Imputation } & \multicolumn{4}{|c|}{ Bayesian } \\
\hline & & & $10 \%$ & $50 \%$ & $65 \%$ & $80 \%$ & $10 \%$ & $50 \%$ & $65 \%$ & $80 \%$ \\
\hline Brier Score & 0.062 & 0.062 & 0.067 & 0.24 & 0.18 & 0.19 & 0.062 & 0.063 & 0.062 & 0.063 \\
\hline$A \cup C^{C}$ & 0.67 & 0.67 & 0.64 & 0.49 & 0.42 & 0.49 & 0.67 & 0.67 & 0.65 & 0.64 \\
\hline
\end{tabular}
observations per DHB.

Table 4 Comparison of Brier Score and Area Under the Curve (AUC) between Bayesian and Multiple Imputation models for the prediction of overseas travel status of campylobacteriosis cases

${ }^{a} n=44,285$

${ }^{\mathrm{b}}$ Four categories of artificially introduced missing data (10\%, $50 \%, 65 \%$ and $80 \%$ missing overseas travel status)

'Area Under the Receiver Operating Characteristic Curve 


\section{Results}

Figure 1 displays the total number of notified Campylobacter cases between 2000 and 2010 which are categorized by the status of overseas travel reporting and annual rate of overseas travels per person in each DHB. Most of the cases reported from Auckland, Waitemata, and Counties Manukau DHBs lack travel information. However, the majority of reported cases and more than $55 \%$ of all travel between 2000 and 2010 originated from residents in these DHBs [27]. As shown in Fig. 1, more than $60 \%$ of all cases come from six DHBs, namely Waitemata (12.8 \%), Canterbury (12.7 \%),
Auckland (10.6 \%), Waikato (9.3 \%), Capital and Coast (8.9 \%), and Counties Manukau (8.7 \%) (Fig. 1).

The number of short term international trips by New Zealanders consistently increased between 2000 and 2010 (bottom panel in Fig. 2). As evident from Fig. 2, total campylobacteriosis notification in New Zealand had been increasing until 2006 except a slight decrease in 2003-2004. After 2006, the total number of notifications declined significantly. The total number of reported travel associated cases and the overall trend of availability of information on travel status for the notified campylobacteriosis cases have declined over time

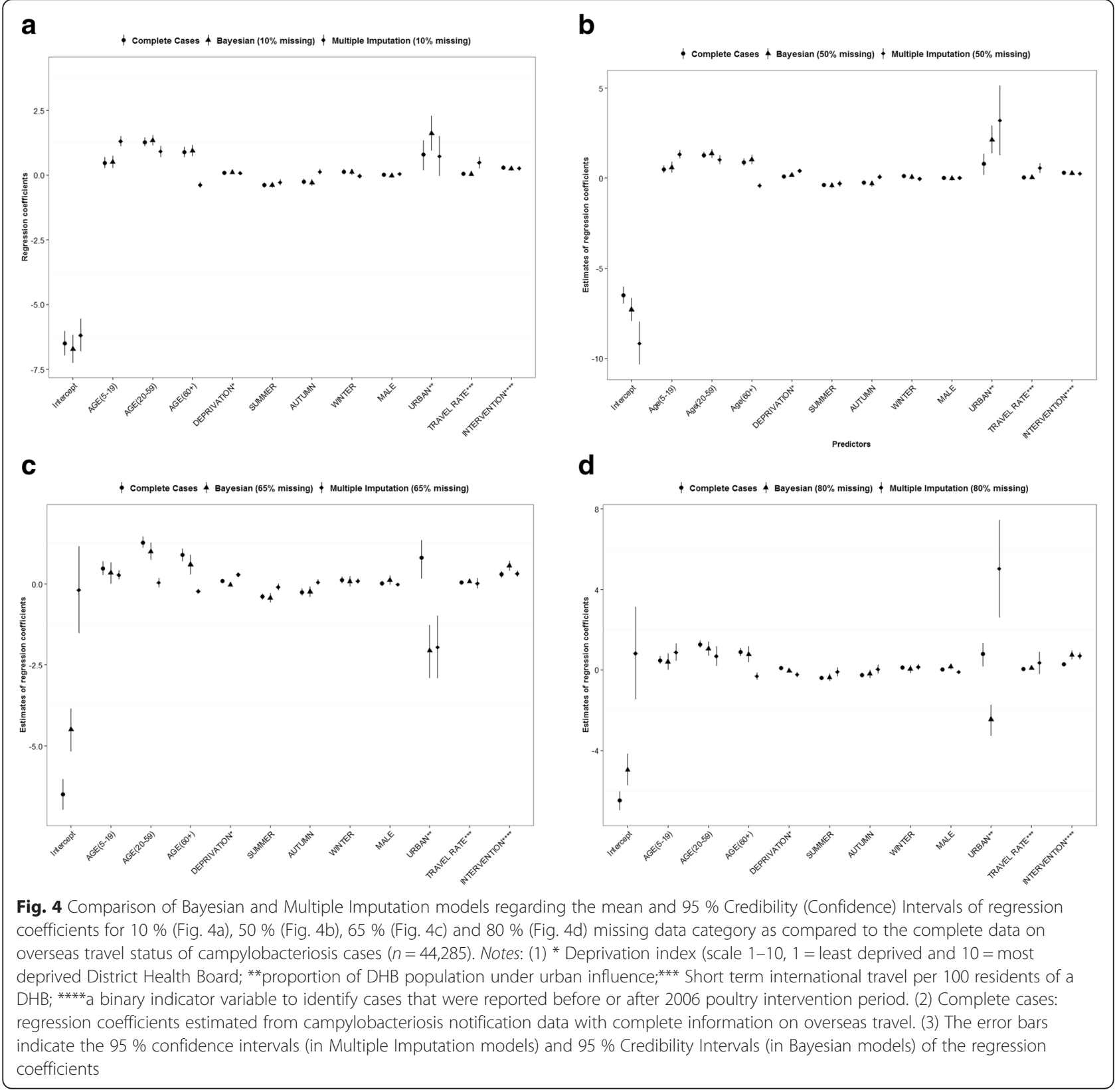


except a slight increase in 2010 (see the middle panel of Fig. 2 and Fig. 3).

Table 3 shows the result of statistically examining the association between predictors and whether or not overseas travel was missing using the original dataset $(n=116,721)$. Majority of the predictors are strongly associated with missingness in overseas travel implying that missingness in the data can be explained by the fully observed variables in the model which supports the MAR assumption (Table 3).

The outcomes of applying MI and BAS models to the datasets with artificially induced missingness is given in Table 4 and Fig. 4. Comparison of BS and AUC to select the best predictive model shows that the BAS model is more robust than $\mathrm{MI}$ as the rate of missingness increases (Table 4). At $10 \%$ MAR, there was no difference between MI and BAS. However at $50 \%, 65 \%$ and $80 \%$ MAR cases, the BAS approach resulted in relatively higher AUC and smaller BS than MI (Table 4). Furthermore, results of the model outputs (i.e., mean and $95 \%$ uncertainty bounds of the regression coefficients) for all categories of missing data as well as the outputs from the complete cases are presented in Fig. 4a, b, c and d. There was no remarkable difference in the regression coefficient estimates across the four categories of artificial missing data. However, most of the regression estimates and $95 \%$ CIs of the BAS model are closer to the values estimated using complete cases as compared to the estimates from the MI model (Fig. 4). This evidence suggests that, the BAS model performs relatively better for a dataset with a high rate of missing values. In addition, no significant difference between the regression coefficient estimates was observed from the Bayesian model fit to original dataset $(n=116721)$ and to the CC dataset $(n=44,285)$ (see Table 5$)$.

The BAS model was applied to the original dataset to estimate the proportion of cases due to overseas travel in each DHB during the period 2008-2010. During this period the number of campylobacteriosis notifications and travel rates were relatively stable. Figure 5 shows the total number of notified campylobacteriosis cases (upper panel) and the estimated proportion of travel related cases as predicted by our model (lower panel). The horizontal dashed line in the bottom panel is drawn to indicate the percent of reported travel associated cases (7\%) among all cases that have provided travel information.

In many of the DHBs with a high rate of campylobacteriosis notification (see upper panel of Fig. 5) and high rate of travel (see bottom panel of Fig. 1), such as Auckland, Counties Manukau and Waitemata, our model predicted a high proportion of campylobacteriosis cases to be associated with overseas travel. For example, the proportion of travel associated cases was higher in Counties Manukau, Auckland and Waitemata DHBs, at 0.34 (0.12,
Table 5 Summary of logistic regression coefficients for the original dataset containing missing observations $(n=116,721)$ and the Complete Cases dataset $(n=44,285)$ using Bayesian models

\begin{tabular}{|c|c|c|c|c|c|c|}
\hline \multirow[t]{2}{*}{ Coefficients } & \multicolumn{2}{|c|}{ Original dataset ${ }^{a}$} & \multicolumn{4}{|c|}{ Complete Cases $^{\mathrm{b}}$} \\
\hline & Mean & $95 \% \mathrm{Cl}^{2}$ & & Mean & $95 \% \mathrm{Cl}$ & \\
\hline Intercept & -6.503 & -6.965 & -6.041 & -6.522 & -6.978 & -6.070 \\
\hline Urban $^{c}$ & 0.804 & 0.231 & 1.377 & 0.834 & 0.297 & 1.414 \\
\hline Deplndex ${ }^{d}$ & 0.091 & 0.063 & 0.119 & 0.091 & 0.063 & 0.120 \\
\hline Travel Rate ${ }^{\mathrm{e}}$ & 0.045 & 0.040 & 0.051 & 0.045 & 0.039 & 0.050 \\
\hline fye $(3-19)$ & 0.473 & 0.262 & 0.683 & 0.476 & 0.270 & 0.680 \\
\hline Age (20-59) & 1.273 & 1.095 & 1.452 & 1.278 & 1.105 & 1.449 \\
\hline Age (60+) & 0.885 & 0.688 & 1.082 & 0.889 & 0.697 & 1.080 \\
\hline Summer & -0.393 & -0.491 & -0.294 & -0.393 & -0.491 & -0.297 \\
\hline Autumn & -0.254 & -0.364 & -0.143 & -0.255 & -0.367 & -0.145 \\
\hline Winter & 0.128 & 0.027 & 0.230 & 0.128 & 0.026 & 0.229 \\
\hline Male & 0.015 & -0.060 & 0.090 & 0.015 & -0.059 & 0.089 \\
\hline Intervention $^{f}$ & 0.288 & 0.200 & 0.377 & 0.287 & 0.199 & 0.377 \\
\hline
\end{tabular}

${ }^{a}$ All campylobacteriosis notifications available for analysis $(n=116,271)$; ${ }^{b}$ campylobacteriosis notifications containing information on overseas travel status $(n=44,285)$. ${ }^{\text {C }}$ Proportion of DHB population under urban influence; ${ }^{\mathrm{d}}$ Deprivation index (scale $0-10,0=$ least deprived and $10=$ most deprived $\mathrm{DHB})$; ${ }^{\mathrm{S}}$ Short term international travel per 100 residents of a $\mathrm{DHB} ;{ }^{\mathrm{f}} \mathrm{A}$ binary indicator variable to identify pre and post 2006 intervention. Age $(<5)$, Spring, and Female sex are reference categories

$0.57), 0.33(0.13,0.55)$ and $0.28(0.10,0.49)$, whereas the lowest proportions were estimated for West Coast, Northland and Tairawhiti at $0.02(0.01,0.06), 0.03(0.01$, $0.08)$ and $0.04(0.01,0.08)$ respectively. Except for Auckland, Counties Manukau, West Coast and Waitemata DHBs, the $95 \% \mathrm{CI}$ of the predicted proportion of travel associated cases included the observed national proportion of travel related cases (horizontal dashed line in bottom panel of Fig. 5). Accordingly, the national estimate and $95 \% \mathrm{CI}$ of the proportion of travel related cases based on our model is $0.16(0.02,0.48)$.

\section{Discussion}

Data gaps in notification data have been a continuous public health challenge for identifying the source of infection and preventing infectious diseases, including campylobacteriosis. The increase of overseas travel by New Zealanders and the established risk of overseas travel for Campylobacter infection emphasize the need to study travel associated illnesses.

A total of 18.3 million short term international trips by New Zealand residents were recorded between 2000 and 2010. Most travel was to the Pacific region, East Asia and North America, while the least travel was recorded for the regions of West and Central Africa and Antarctica. This is in agreement with previous reports that New Zealanders travel to more than 150 countries, of which countries in the Pacific region and North 

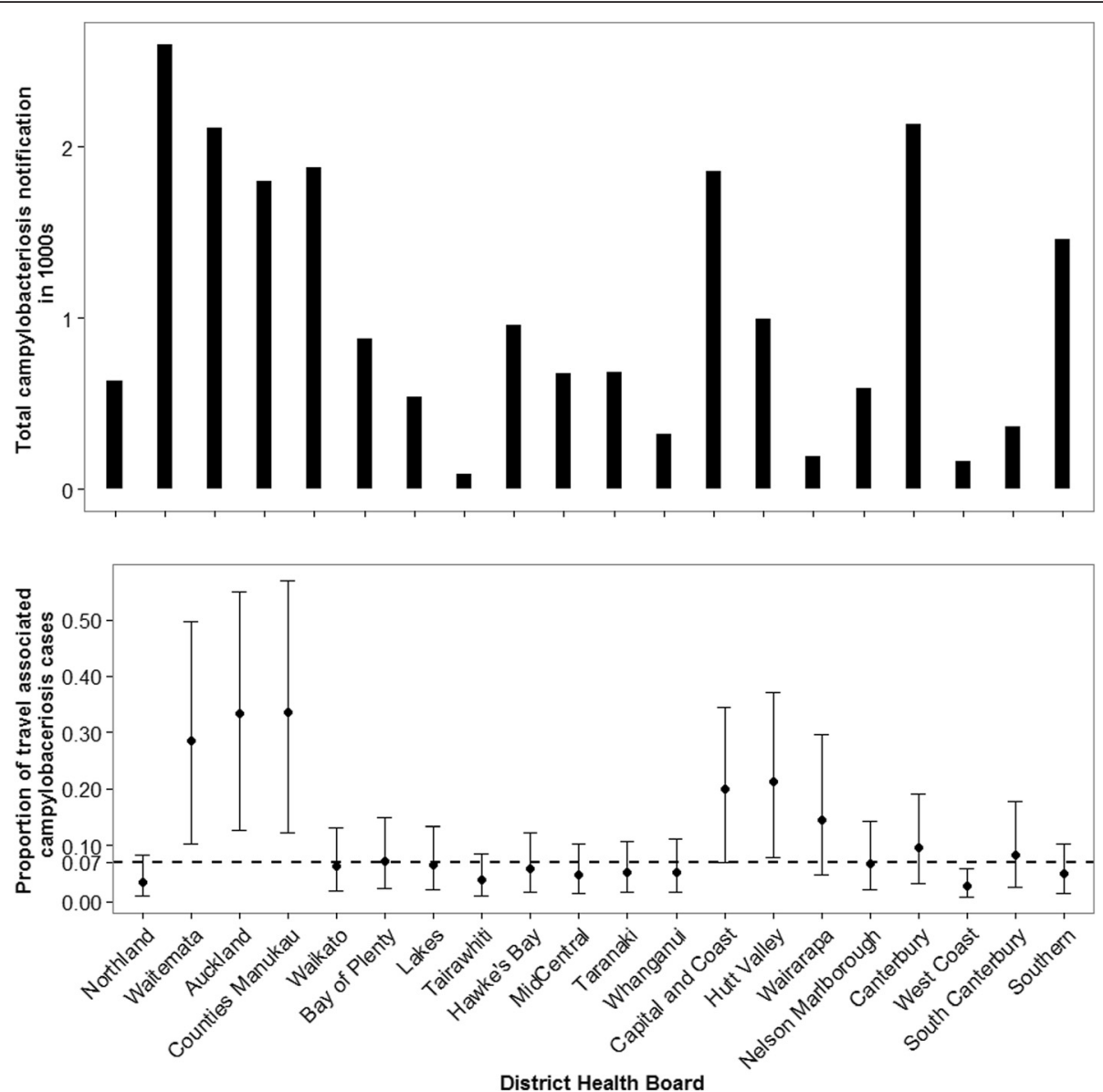

Fig. 5 The total number of campylobacteriosis notification (upper panel) and the proportion of travel related cases predicted by the Bayesian model (lower panel) for each DHB of New Zealand (2008-2010). Notes: (1) Bottom panel: proportion of travel related cases predicted by the Bayesian model. The error bars are $95 \%$ Credibility Intervals of the proportion of overseas travel. (2) The dashed horizontal line is the proportion of travel related campylobacteriosis cases for which travel history is available nationally (7 \%)

America are the most popular destinations [6]. In the meantime, international travel has been increasing in New Zealand since 2004 (see Fig. 2). In contrast, a substantial reduction of incidence of notified campylobacteriosis cases occurred after 2006 (Fig. 2). The significant changes in notifications post 2006 were believed to be the result of interventions targeting poultry [2]. Despite this overall decline in notifications of campylobacteriosis in New Zealand, the change attributable to cases associated with overseas travel is not well understood. Although the outbound travel rate of New Zealand residents has been increasing, we noticed a decline in notified travel associated cases throughout the study period except a slight increase in 2010 (middle panel of Fig. 2). This could be due to the corresponding decrease in reporting of travel information for the cases throughout the study period (Fig. 3) that may have confounded conclusions on the origin of the disease.

In addition, there is a consistently low reporting rate of detailed travel information in urban areas of New Zealand such as in Auckland and Wellington regions. A case control study in the New Zealand regions with high notifications rates, including Auckland region, suggests that recent overseas travel was a significant risk factor for the occurrence of campylobacteriosis in this region [28].

The majority (62\%) of campylobacteriosis case reports in New Zealand lack travel history during the incubation period prior to disease. The level of completeness of travel history for notified cases has been a challenging task as is reported by some other studies [29-31]. It is therefore necessary to estimate travel associated cases based on imperfect data.

Among the total number of notifications with known travel history, only 3107 (7 \% of notifications with known travel status) had travelled overseas during the incubation of the disease. As New Zealanders are prolific travelers, this proportion of cases may underestimate the true contribution of travel as a risk factor for campylobacteriosis in New Zealand. For this reason, modelbased methods such as MI and BAS can be useful to fill 
the data gaps, using covariates that predict overseas travel. The use of BAS and MI methods provides a methodology to calculate uncertainty bounds around the estimates of travel associated cases. The degree of uncertainty of the predicted proportion of travel associated cases can be attributed to variation in the risk of travel associated illnesses among individuals that have different covariate values. Such variation in the risk of campylobacteriosis with respect to age, sex and season is in agreement with previous reports in literature [5, 32].

The BAS model resulted in an estimate of the national proportion of notifications due to overseas travel of $16 \%$, a higher value compared to $7 \%$ estimate using only known values. Similar or higher rates of travel related campylobacteriosis have been reported in other developed countries such as in Canada (21.6 \%) [33], England (17 \%) [29], USA (18 \%) [34], Denmark (18 \%) [35] and Switzerland (46.1\%) [36].

Our model predicted a high proportion of travel associated cases in major urban areas of New Zealand, such as in Auckland, Counties Manukau and Waitemata DHBs. This could be due to high rates of travel of their residents to the Pacific Islands and South East Asia regions, which is partially driven by the comparatively high proportion of Asian ethnicity (23.8 \%) and Pacific Peoples $(14,6 \%)$ in the Auckland region $[6,27]$. It has been previously established that individuals traveling to these world regions are at a higher risk of travel associated illnesses, including campylobacteriosis [37]. On the other hand, the DHBs with a smaller proportion of modelpredicted travel related cases (e.g., Northland, West Coast and Tairawhiti) are those with a lower outbound travel rate.

If the MAR assumption holds, which is usually difficult to achieve, our Bayesian model provides a plausible way for predicting missing overseas travel of campylobacteriosis cases [20]. It is also important to note that any other missing data analysis approaches require assumptions that are just as difficult to justify [11]. At the same time, the BAS procedure should not be viewed as the 'gold standard' for filling data gaps for every situation, although it offers a flexible approach for data augmentation. Priors can be enhanced if data regarding association of risk factor-outcome become available. In addition, the Bayesian model specification can be modified if the missing data mechanism is non-ignorable and the missingness model can be verifiable [38].

Better notification reporting, particularly for areas with high outbound travel and high notification of cases such as in highly urban areas can improve our understanding of the epidemiology of travel associated campylobacteriosis in New Zealand. However, reporting completeness is limited by the resources available in each DHB. Use of alternative data collection approaches such as web based applications, cross tabulation of Customs data with EpiSurv data, and creating awareness in the population regarding the importance of the information for the public health databases may improve reporting completeness. Although the emphasis in this report is on predicting travel information of Campylobacter cases in New Zealand, the method can be implemented for other diseases of public health significance which have similar data gaps.

\section{Conclusion}

The common challenge of data gaps regarding risk factors for campylobacteriosis suggests the use of modelbased approaches for estimating missing values. Filling data gaps is particularly important for regions with a high rate of incomplete data. The Bayesian modelling approach offers a flexible alternative for data augmentation particularly when the missing rate is very high.

\section{Additional files}

Additional file 1: The description of explanatory variables used for predicting overseas travel status of campylobacteriosis notification in New Zealand $[2,27,39]$. (DOCX $16.3 \mathrm{~kb}$ )

Additional file 2: JAGS code for the Bayesian Hierarchical model $[22,40]$. (DOCX $17.5 \mathrm{~kb})$

Additional file 3: Number of campylobacteriosis notifications and overseas travels in New Zealand District Health Boards (DHBs) (20002010). (XLSX $11 \mathrm{~kb}$ )

\section{Acknowledgments}

The authors would like to thank the Institute of Environmental Science \& Research Ltd (ESR) and the Ministry of Health for providing parts of the data and information. However, the analyses, conclusions, opinions and statements expressed herein are those of the authors, and not necessarily those of the ESR or the Ministry of Health.

\section{Funding}

This work was supported by the NIH Ruth L. Kirschstein National Research Service Award Institutional Training Grant T32 RR023916 and T32 OD010423.

\section{Availability of data and materials}

Summary of the dataset supporting the findings of this article are summarized in Table 1 and in Additional file 3. The authors are unable to provide direct access to the raw data used. However requests for the data can be made from following sources: (1) Travel data can requested from Statistics New Zealand (www.stats.govt.nz) and (2) Public health surveillance data can be requested from ESR (https://surv.esr.cri.nz).

\section{Authors' contributions}

$\mathrm{BH}, \mathrm{RL}, \mathrm{DD}, \mathrm{EA}$ : conceived and designed the study; EA, DD performed the data analysis; $\mathrm{EA}, \mathrm{BH}, \mathrm{RP}, \mathrm{RL}$ and $\mathrm{DD}$ were involved in drafting and reviewing the Manuscript. All authors read and approved the final version of the manuscript.

\section{Competing interests}

The authors declare that they have no competing interests.

Consent for publication

Not applicable. 


\section{Ethics approval and consent to participate}

This study has been reviewed and approved by the New Zealand Southern Health and Disability Ethics Committee (Ethics Reference: MEC/12/EXP/029/ AM03). The study has also been approved by the Institutional Review Board (IRB) at UW-Madison (IRB no. 2013-0072).

\section{Author details}

'Department of Medical Sciences, School of Veterinary Medicine, University of Wisconsin-Madison, Madison, USA. ${ }^{2}$ Institute of Environmental Science and Research, Christchurch, New Zealand.

\section{Received: 13 September 2015 Accepted: 16 August 2016} Published online: 06 September 2016

\section{References}

1. Baker MG, Sneyd E, Wilson NA. Is the major increase in notified campylobacteriosis in New Zealand real? Epidemiol Infect. 2007;135:163-70.

2. Sears A, Baker MG, Wilson N, Marshall J, Muellner P, Campbell DM, et al. Marked campylobacteriosis decline after interventions aimed at poultry. New Zealand Emerg Infect Dis. 2011;17:1007-15.

3. Mullner P, Shadbolt T, Collins-Emerson JM, Midwinter AC, Spencer SEF, Marshall J, et al. Molecular and spatial epidemiology of human campylobacteriosis: source association and genotype-related risk factors. Epidemiol Infect. 2010;138:1372-83.

4. Horn BJ, Lake RJ. Incubation period for campylobacteriosis and its importance in the estimation of incidence related to travel. Euro Surveill Bull Eur Sur Mal Transm Eur Commun Dis Bull. 2013;18:1-6.

5. Ekdahl K, Andersson Y. Regional risks and seasonality in travel-associated campylobacteriosis. BMC Infect Dis. 2004;4:54.

6. Outbound Travel by New Zealand Residents [Internet]. The Ministry of Tourism; 2009 [cited 2015 Jul 29]. Available from: www.tourismresearch. govt.nz. Accessed 29 July 2015

7. van Buuren S. Flexible imputation of missing data. Boca Raton: CRC Press; 2012.

8. Gelman A, Hill J. Data Analysis Using Regression and Multilevel/Hierarchica Models. Cambridge: University Press; 2006.

9. EpiSurv. Public Health Surveillance. Available from: https://surv.esr.cri.nz/ episurv/index.php. Accessed 24 Apr 2014.

10. Rubin DB. Inference and missing data. Biometrika. 1976:63:581-92.

11. Enders CK. Applied missing data analysis. New York: Guilford Press; 2010

12. Schafer JL. Multiple imputation: a primer. Stat Methods Med Res. 1999:8:3-15

13. Harel $\mathrm{O}$, Zhou $\mathrm{X}-\mathrm{H}$. Multiple imputation: review of theory, implementation and software. Stat Med. 2007;26:3057-77.

14. Van Buuren S, Boshuizen HC, Knook DL. Multiple imputation of missing blood pressure covariates in survival analysis. Stat Med. 1999;18:681-94.

15. Rubin DB. Multiple imputation for nonresponse in surveys. Hoboken: John Wiley \& Sons, Inc.; 1987.

16. Horton NJ, Lipsitz SR. Multiple imputation in practice: comparison of software packages for regression models with missing variables. Am Stat. 2001:55:244-54.

17. Liu M, Taylor JM, Belin TR. Multiple imputation and posterior simulation for multivariate missing data in longitudinal studies. Biometrics. 2000:56:1157-63.

18. Jackman S. Estimation and inference via Bayesian simulation: an introduction to Markov Chain Monte Carlo. Am J Polit Sci. 2000;44:375-404.

19. Horton NJ, Kleinman KP. Much ado about nothing: A comparison of missing data methods and software to fit incomplete data regression models. Am Stat. 2007;61:79-90

20. Mason A, Best N, Richardson S, Plewis I. Strategy for modelling non-random missing data mechanisms in observational studies using Bayesian methods. 2010. Available from: http://eprints.ncrm.ac.uk/1776/. Accessed 23 Jul 2014

21. Mason A, Best N, Plewis I, Richardson S. Insights into the use of Bayesian models for informative missing data. Imperial College London; 2010. Available from: www.bias-project.org.uk

22. Plummer M, Others. JAGS: A program for analysis of Bayesian graphical models using Gibbs sampling. Proc. 3rd Int. Workshop Distrib. Stat. Comput. Technische Universit at Wien; 2003. p. 125. Available from: http://www.ci. tuwien.ac.at/Conferences/DSC-2003/Drafts/Plummer.pdf. Accessed 9 Jun 2015
23. Gelman A, Carlin JB, Stern HS, Dunson DB, Vehtari A, Rubin DB. Bayesian Data Analysis, Third Edition. 3rd ed. Boca Raton: Chapman and Hall/CRC; 2013.

24. Brier GW. Verification of forecasts expressed in terms of probability. Mon Weather Rev. 1950;78:1-3.

25. Steyerberg E. Clinical prediction models: A practical approach to development, validation, and updating. Springer-Verlag New York: Springer Science \& Business Media; 2008.

26. Cleves MA. Comparative assessment of three common algorithms for estimating the variance of the area under the nonparametric receiver operating characteristic curve. Stata J. 2002:3:280-9.

27. Statistics New Zealand. Home - Statistics New Zealand. Available from: http://www.stats.govt.nz/. Accessed 10 Apr 2014

28. Eberhart-Phillips J, Walker N, Garrett N, Bell D, Sinclair D, Rainger W, et al. Campylobacteriosis in New Zealand: results of a case-control study. J Epidemiol Community Health. 1997;51:686-91.

29. Zenner D, Gillespie I. Travel-associated Salmonella and Campylobacter gastroenteritis in England: estimation of under-ascertainment through national laboratory surveillance. J Travel Med. 2011;18:414-7.

30. Guzman-Herrador B, Vold L, Nygard K. Surveillance of travel-associated gastrointestinal infections in Norway, 2009-2010: are they all actually imported? Euro Surveill Bull Eur Sur Mal Transm Eur Commun Dis Bull. 2012;17:20294

31. Lake R, Horn B, Ball A, New Zealand, Ministry of Agriculture and Forestry, MAF Biosecurity New Zealand, et al. Campylobacter in food and the environment, examining the link with public health pathway attribution. Wellington: Ministry of Agriculture and Forestry; 2011.

32. Unicomb LE, Dalton CB, Gilbert GL, Becker NG, Patel MS. Age-specific risk factors for sporadic Campylobacter infection in regional Australia. Foodborne Pathog Dis. 2008:5:79-85.

33. Ravel A, Nesbitt A, Marshall B, Sittler N, Pollari F. Description and burden of travel-related cases caused by enteropathogens reported in a Canadian community. J Travel Med. 2011;18:8-19.

34. Ricotta EE, Palmer A, Wymore K, Clogher P, Oosmanally N, Robinson T, et al. Epidemiology and antimicrobial resistance of international travel-associated Campylobacter infections in the United States, 2005-2011. Am J Public Health. 2014:104:108-14.

35. Neimann J, Engberg J, Mølbak K, Wegener HC. A case-control study of risk factors for sporadic campylobacter infections in Denmark. Epidemiol Infect. 2003;130:353-66.

36. Schorr D, Schmid H, Rieder HL, Baumgartner A, Vorkauf H, Burnens A. Risk factors for Campylobacter enteritis in Switzerland. Zentralblatt Für Hyg Umweltmed Int J Hyg Environ Med. 1994;196:327-37.

37. Dupont HL. Systematic review: prevention of travellers' diarrhoea: Systematic review: prevention of travelers' diarrhea. Aliment Pharmacol Ther 2008;27:741-51.

38. Mason A, Richardson S, Best N. Two-pronged Strategy for Using DIC to Compare Selection Models with Non-Ignorable Missing Responses. Bayesian Anal. 2012;7:109-46.

39. Salmond C, Crampton P, Atkinson J. NZDep2006: New Zealand Index of Deprivation. 2007

40. Plummer M. rjags: Bayesian graphical models using MCMC. R package version 3-10. 2013. Available from: http://CRAN.R-project.org/package=rjags.

\section{Submit your next manuscript to BioMed Central and we will help you at every step:}

- We accept pre-submission inquiries

- Our selector tool helps you to find the most relevant journal

- We provide round the clock customer support

- Convenient online submission

- Thorough peer review

- Inclusion in PubMed and all major indexing services

- Maximum visibility for your research

Submit your manuscript at www.biomedcentral.com/submit 